\title{
Nach demm und wir üch jetz geschriben hant von der getat. Kriegsordnungen, Chroniken, Berichte und Briefe aus dem Feld. Quellen zum Ragazer Feldzug 1446 im Alten Zürichkrieg
}

\section{Letters, Reports and Chronicles. Sources for the 1446 Confederate Campaign in the Old Zurich War}

Heinrich Speich / heinrich.speich@ibid.ch

Ústav pomocných věd historických a archivnictví, Filozofická fakulta, Masarykova univerzita, Brno, CZ

\begin{abstract}
In February 1446 a group of about 1100 confederate soldiers went on a short campaign to the neighbouring county of Sargans. The campaign can function as an example for the wide variety of texts produced alongside military actions in the $15^{\text {th }}$ century. Therefore we find the protocols of the preparatory federal diets that agree the number of soldiers and discuss plans. As the campaign goes on, there are two letters of the Lucerne captain Cloos to his authority, giving short information on encounters and claiming money and reinforcements. On the Habsburg side, we have written evidence for the raising and equipment of troops in Vorarlberg as well as for the financial efforts for the counter-campaign. After the campaign and the victorious battle at Ragaz, confederate authors composed tales of the battle: one being the report of the Bernese captain to his authority, the other was the chancellor of Schwyz Hans Fründ with his quasi-offical chronicle of the Old Zürich war. Soon after, the annual commemoration of the battle of Ragaz (6 March 1446) was extended to all minor battles of the Confederates. The dead and mortally in all battles were registered in the parish-registers under the day of St Fridolin, the patron saint of Glarus (6 March). As a result of these texts, the campaign has subsequently been considered a success for the Confederates.
\end{abstract}

\section{Keywords}

Swiss Confederation; Warfare; Campaign; Habsburg; Sargans; Rhine; Commemoration; 1446

Der vorliegende Aufsatz entstand im Rahmen des von der Grantová agentura České republiky/Czech Science Foundation geförderten Forschungsprojektes GX19-28415X „From Performativity to Institutionalization: Handling Conflict in the Late Middle Ages (Strategies, Agents, Communication)." 
Im Februar 1446 zogen etwa 1100 Eidgenossen aus den Städten Bern, Luzern und den Innerschweizer Länderorten ins Sarganserland, einem Nebenschauplatz des Alten Zürichkriegs. ${ }^{1}$ Der Feldzug dauerte nur rund drei Wochen. Mitte März zogen die Eidgenossen nach einer gewonnenen Schlacht wieder ab und überliessen das Land wieder den habsburgischen Dienstleuten.

\section{Eidgenossen, Schriftlichkeit und Krieg}

Dieser merkwürdige kurze Feldzug ist aus verschiedenen Gründen einer näheren Betrachtung wert ${ }^{2}$. Einerseits war es die letzte grössere Auseinandersetzung am Ende des Alten Zürichkrieges (1436-1446/50), andererseits fand gerade dieser kurze Feldzug reichen Niederschlag in den Quellen der Streitparteien und in der Chronistik. ${ }^{3}$ Die schriftlichen Quellen zur Überlieferung für den Ragazer Feldzug von 1446 dürfen innerhalb des gut erforschten spätmittelalterlichen Schweizer Kriegswesen als ausgesprochen dicht bezeichnet werden ${ }^{4}$. Anhand dieses reichen Quellenmaterials kann gezeigt werden, welche Quellengattungen in diesem langandauernden Konflikt produziert und wie diese angewendet wurden. Der Hauptfokus liegt auf den am Alten Zürichkrieg besonders intensiv beteiligten eidgenössischen Länderorten. Diese entdeckten gerade aufgrund der andauernden Konfliktsituation ihren Bedarf an Schriftlichkeit.

1 Einführend zum Alten Zürichkrieg siehe Niederstätter, Alois: Der alte Zürichkrieg. Studien zum österreichisch-eidgenössischen Konflikt sowie zur Politik König Friedrichs III. in den Jahren 1440 bis 1446. Forschungen zur Kaiser- und Papstgeschichte des Mittelalters. Beihefte zu J. F. Böhmer. Regesta Imperii 14. Wien - Köln - Weimar 1995; Ein „Bruderkrieg“ macht Geschichte. Neue Zugänge zum Alten Zürichkrieg. Hg. von P. Niederhäuser und Ch. Sieber. Mitteilungen der Antiquarischen Gesellschaft Zürich 73. Zürich 2006; Stettler, Bernhard: Die Eidgenossenschaft im 15. Jahrhundert. Auf der Suche nach einem gemeinsamen Nenner. Menziken 2004.

2 Zum Ragazer Feldzug und seinen sozialhistorischen Fragestellungen siehe Speich, Heinrich: Netzwerke im Stresstest. Netzwerkdynamik in Kriegszeiten am Beispiel des Ragazer-Feldzuges 1446. In: Die Grenzen des Netzwerks. Hg. von K. Hitzbleck und K. Hübner. Ostfildern 2014, S. 197-222. Die lokalhistorische Perspektive ist aufgearbeitet bei Rigendinger, Fritz: Das Sarganserland im Spätmittelalter. Lokale Herrschaften, die Grafschaft Sargans und die Grafen von Werdenberg-Sargans. Zürich 2007. Zur allgemeinen militärhistorischen Relevanz vgl. Bachrach, Bernard S. - Bachrach, David S.: Warfare in Medieval Europe c. 400 - c. 1453, S. 175-179.

3 Zur Quellenproduktion eidgenössischer Feldzüge allgemein bei Landolt, Oliver: Switzerland. In: The Oxford Encyclopedia of Medieval Warfare and Military Technology. Hg. von C. Rogers. Vol. 3. Oxford 2010, S. 324-335. Zur Chronistik: Stettler, Bernhard: Tschudis Bild vom Alten Zürichkrieg und dessen Platz in der Historiographie. In: Aegidius Tschudi, Chronicon Helveticum. Bd. 12. Bearb. von B. Stettler. Bern 1998, S. $1 *-121 *$.

4 Archivbezeichnungen StA=Staatsarchiv. Dazu das Kantonskürzel ZH=Zürich, BE=Bern, LU=Luzern, $\mathrm{UR}=\mathrm{Uri}, \mathrm{SZ}=\mathrm{Schwyz}, \mathrm{OW}=$ Obwalden, $\mathrm{ZG}=\mathrm{Zug}, \mathrm{GL}=\mathrm{Glarus}, \mathrm{SO}=$ Solothurn. Weitere Archive sind ausgeschrieben. Traditionelle Werke zur spätmittelalterlichen Kriegsgeschichte, denen hier aus moderner sozialhistorischer Perspektive weitgehend widersprochen werden muss: Dürr, Emil: Die Politik der Eidgenossen im 14. und 15. Jahrhundert. In: Schweizer Kriegsgeschichte. Heft 4. Bern 1933; Wackernagel, Hans Georg: Kriegsbräuche in der mittelalterlichen Eidgenossenschaft. Basel 1934; Schaufelberger, Walter: Der Alte Schweizer und sein Krieg. Studien zur Kriegführung vornehmlich im 15. Jahrhundert. Frauenfeld 1987³ (1. Auflage Zürich 1952). 


\section{Die Macht der Feder}

Im Kontext militärischer Aktionen von Städten wurde es seit dem 14. Jahrhundert gebräuchlich, entlang einer Konfliktlage spezifisches Gebrauchsschriftgut anzufertigen. Diese Praxis war in den weitgehend illiteraten Abläufen ländlicher Orte der Eidgenossenschaft bis Mitte des 15. Jahrhunderts noch nicht etabliert. Die Überlieferung zu den frühen Kriegen der Eidgenossenschaft stützt sich bis weit ins 15. Jahrhundert einseitig auf die städtische und geistliche Quellenproduktion ab. Gerade der Alte Zürichkrieg beförderte insbesondere in den eidgenössischen Länderorten die Schriftlichkeit ganz entscheidend. Am Augenfälligsten ist dies an der Berufung des Luzerner Bürgers Hans Fründ als Schreiber an die Kanzlei des Länderortes Schwyz im Jahr 1437. ${ }^{5}$ Der Länderort war sich der Bedeutung adäquater Schriftproduktion im diplomatischen Dauerkampf mit der Stadt Zürich bereits in der ersten Konfliktphase ab 1436 bewusst. Hans Fründ war seit 1427 Unterschreiber in Luzern und wechselte als erster auswärtiger Schreiber und Notar nach Schwyz. Damit zog Schwyz auf dem diplomatischen Parkett mit Zürichs versiertem Schreiber und kaiserlichem Notar Michael Graf gleich. ${ }^{6}$ Aber Fründ konnte mehr: er war ein begnadeter Tagebuchschreiber und hinterliess eine Chronik, die uns heute als eine der zentralen Informationsquellen zum Alten Zürichkrieg dient. ${ }^{7}$

\section{Vorbereitung des Feldzuges}

Zum Feldzug von 1446 besitzen wir die Traktandenliste der vorbereitenden Tagsatzung in Luzern vom 25. Januar 1446. ${ }^{8}$ Dort wurde den Appenzellern zugesagt, sie im Rheintal, am Eschenberg zu unterstützen. Es wurde vereinbart, dass sich Appenzeller und Eidgenossen im Rheintal vereinen sollten, um habsburgische Kräfte in Vorarlberg anzugreifen. Dazu wurde im Vorfeld an der eidgenössischen Tagsatzung geklärt, wie das Aufgebot zusammen zu stellen sei. Das Aufgebot erfolgte separat in den einzelnen Orten, gemeinsame Aufgebote gab es nicht. Die einzelnen Orte mussten ihre Verbündeten jeweils eigens zur Kriegsteilnahme auffordern, sog. „mahnen“. ${ }^{9}$ Aus anderen zeitgleichen Konflikten

5 Sieber, Christian: «Unfreundliche» Briefe, Kriegserklärungen und Friedensverträge - Der Alte Zürichkrieg (14361450) im Spiegel der Biographie von Landschreiber Hans Fründ. Jahrbuch des Historischen Vereins des Kantons Schwyz 98, 2006, S. 11-37. Stettler, Bernhard: Zur Überlieferung von Hans Fründs. Darstellung des Alten Zürichkriegs. In: Aegidius Tschudi, Chronicon Helveticum. Bd. 12, S. 122*-134*.

6 Zu Michael Graf auch genannt Stebler siehe Sieber, Christian: Politik, Verwaltung und Archiv. Aufstieg und Fall der „kaiserlichen“ Stadt Zürich zwischen 1430 und 1450 im Spiegel der Biographien von Bürgermeister Rudolf Stüssi und Stadtschreiber Michael Stebler. Zürcher Taschenbuch 127, 2007, S. 1-30.

7 Stiftsbibliothek St. Gallen Cod. Sang. 644, ed. von Kind, Christian Emanuel: Die Chronik des Hans Fründ, Chur 1870. Beschreibung in Scarpatetti, Beat Matthias von: Die Handschriften der Stiftsbibliothek St. Gallen, Bd. 1: Abt. IV: Codices 547-669: Hagiographia, Historica, Geographica, 8.-18. Jahrhundert. Wiesbaden 2003, S. 271-273.

8 Amtliche Sammlung der älteren Eidgenössischen Abschiede (=EA). Bd. 2. Hg. von P. A. von Segesser. Luzern 1863. Bd. 2, S. 197, Nr. 296h: „Die von Appenzell begehren einen Zuzug an den Eschinberg, da der Rhein gegenwärtig klein sei.“

9 Im März 1446 erfolgten weitere Aufgebote Luzerns an den Rhein und nach Pfäffikon am Zürichsee; dort sind die entsprechenden Reisrödel erhalten im Staatsarchiv Luzern (=StALU), Urk 231/3340. Zur 


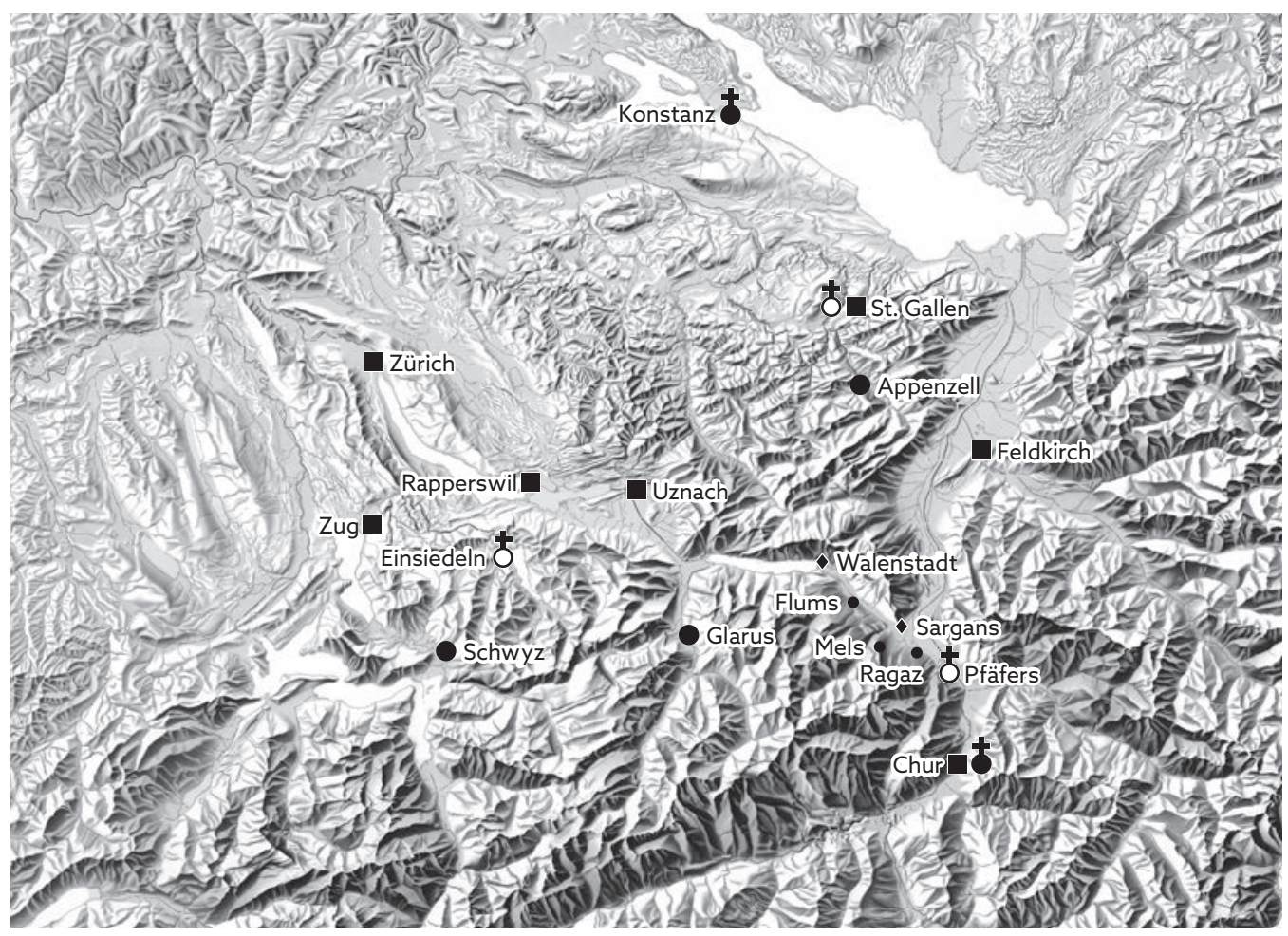

kennen wir Aufgebotsrödel und recht präzise Pläne für Feldzüge und das Aufgebotswesen. vVor allem wenn städtische Kontingente beteiligt waren, sind die Vorbereitungen häufiger schriftlich fassbar. ${ }^{10}$ Als allgemein gültige Kriegsordnung der Eidgenossen für das Verhalten von Bewaffneten darf der sogenannte Sempacher Brief von 1393 gelten. ${ }^{11}$ Dieser war wohl in der Zeit des Alten Zürichkrieges den Hauptleuten noch bekannt. ${ }^{12}$ Die Originale sind jedenfalls in den Archiven der alten eidgenössischen Orte vorhanden. ${ }^{13}$

Mahnung siehe Senn, Hans: Militärwesen. In: Historisches Lexikon der Schweiz, URL vom 02.12.2018: http://www.hls-dhs-dss.ch/textes/d/D24620.php.

10 Häne, Johannes: Militärisches aus dem Alten Zürichkrieg. Zur Entwicklungsgeschichte der Infanterie. Zürich 1928. Häne legte eine Zürcher Aufgebotsliste von 1443 und eine Kriegsordnung für einen Zug von Zürchern nach Diessenhofen von 1444 vor. Laut dem Regest zur Urkunde (oder Abschrift) im Staatsarchiv Zürich (=StAZH), C I, Nr. 1760 handelte es sich um Vorbereitungen zu einem Zug im Winter 1445 oder 1446, der wohl von Hans von Rechberg hätte kommandiert werden sollen. Zur Quellenproduktion allgemein vgl. StALU, URK 231/3340, respektive URK 231/3389 bis URK 231/3394.

11 EA 1, Anhang Nr. 41, S. 327-329: Stettler, Bernhard: Der Sempacherbrief von 1393. Ein verkanntes Dokument aus der älteren Schweizergeschichte. Schweizerische Zeitschrift für Geschichte 35, 1985, S. 1-20.

12 Eine vergleichbare Kriegsordnung ist aus Zürich 1444 erhalten, StAZH, C I, Nr. 1759, ediert bei Häne, J.: Militärisches. Zur Gültigkeit von allgemeinen Regeln im Alten Zürichkrieg siehe Landolt, Oliver: „Wider christenliche ordnung und kriegsbruch ... ". Schwyzerische und eidgenössische Kriegsverbrechen im Spätmittelaltern. Forschungen zur Rechtsarchäologie und rechtlichen Volkskunde 22, 2005, S. 91-121.

13 Nach Archivsignaturschlüssel Anm. 4: StAZH, C I, Nr. 377; StaBE: Fach F, Sempacherbrief 1393.07.10; StALU, URK 45/964; StAUR, AA-100-4, Abschrift des 17. Jh., im Chartular von Landammann Karl Ema- 
Wie sich die Aufgebote sammelten und welche weiteren Absprachen getroffen wurden, ist an unserem Feldzug nur chronikalisch überliefert. Die Chronik von Hans Fründ ist besonders detailliert und damit -zumindest in diesem Fall- glaubwürdig. Fründ berichtet, die Aufgebote der Orte hätten sich in Uznach gesammelt. Uznach war seit 1437 als Pfand in den Händen von Glarus und Schwyz. Absprachen seien mit den Appenzellern, einer locker assoziierten ländlichen Kommune im Hinterland von St. Gallen getroffen worden. Die Appenzeller waren im frühen 15. Jahrhundert öfter über die Bergkette gestiegen und hatten im habsburgisch dominierten Alpenrheintal ihr Unwesen getrieben und auch dieses Mal soll sogar die Initiative zum Feldzug von den Appenzellern ausgegangen sein. Um es vorwegzunehmen: die Appenzeller kamen nicht und ihr Archiv schweigt dazu. Es sammelten sich in Uznach zwischen 1.000 und 1.200 Eidgenossen, das ist eine plausible und in den Quellen übereinstimmend genannte Zahl. ${ }^{14}$ Etwa ein Drittel davon waren Glarner, die mit ihrem Landesbanner erschienen. Dazu noch jeweils ein paar hundert aus Schwyz, Uri und Unterwalden und je etwa 50 aus den Städten Luzern und Bern. Letztere beiden sind für uns wichtig. Der Luzerner Hauptmann schrieb zwei Briefe aus dem Feld, in denen er um Anweisungen bat, der Berner Hauptmann verfasste einen ausführlichen Bericht, der die Basis der Chronik des Bendicht Tschachtlan bildete. Wir stellen fest: Schriftlichkeit im Kriegsalltag war bis dahin weitestgehend eine Domäne der Städte.

\section{Verlauf des Feldzuges}

Über den weiteren Verlauf des Zuges sind wir wiederum aus den Chroniken von Fründ und Tschachtlan bestens unterrichtet: die Eidgenossen fuhren per Schiff bis Quarten und zogen über die Anhöhe der Raischiben. ${ }^{15}$ Dort befand sich die Letzi, eine Landmauer oder verstärkter Hag, welcher als sichtbare Grenze zum Sarganserland diente. ${ }^{16}$ Etwas nach dieser Grenze stellten sich rund hundert Mann aus der österreichischen Garnison in Walenstadt zum Kampf. ${ }^{17}$ Man darf davon ausgehen, dass diese die Aussichtslosigkeit

nuel Bessler von Wettingen; StASZ, Urk Nr. 249: Kopie des 15. Jahrhunderts, Abschrift des Luzerner Exemplars, wohl nach Brand; StAOW, Urk 01.0188; Bürgerarchiv Zug, Nr. 70; Landesarchiv GL, AG III.51.2; StASO, Kopienbuch 1479-1484 (ehemals Copiae rot VIII), S. 441-443, nach den Urkundenabschriften.

14 Aegidius Tschudi, Chronicon Helveticum. Bd. 11. Bearb. von B. Settler. Bern 1996, S. 416. und StALU, Urk $231 / 3391$.

15 Am ausführlichsten ist hier wiederum die Tschudi-Chronik, die zahlreiche Konjekturen des Chronisten enthält, siehe dazu Stettler, Bernhard: Chronicon Helveticum Aegidius Tschudi. Hilfsmittel-Bd. 3; TschudiVademecum: Annäherungen an Aegidius Tschudi und sein „Chronikon Helveticum.“ Bearb. von B. Stettler. Bern 2001; Stettler, Bernhard: Die Historiographie des Alten Zürichkriegs (15.-19. Jahrhundert). In: Ein „Bruderkrieg“. Hg. von P. Niederhäuser - Ch. Sieber, S. 23-42; Feller, Richard - Bonjour, Edgar: Geschichtsschreibung der Schweiz. Bd. 1. Basel - Stuttgart 1979², S. 263-276. Tschudi kannte die Texte des Berner Hauptmanns in der Tschachtlan-Chronik und die Chroniken von Fründ und Brennwald. Die Luzerner Briefe und die Klingenberger Chronik dürfen als Korrektive zu Tschudi angesehen werden.

16 Zu den Letzinen siehe Meyer, Werner: Letzimauern und Landwehren im Spätmittelalter. In: Stadt- und Landmauern. Bd. 1. Beiträge zum Stand der Forschung. Bearb. von B. Siegel. Zürich 1995, S. 107-115.

17 StALU, Urk 231/3390, Brief vom 25. Februar 1446 an Luzern: Uf mitwuchen sind wir in das ober lant komen über die letzi an schaden, wan man vant nieman an der letzi. Und als man uber in kann, do kament unser vigent 
des Kampfes bereits von vornherein gekannt hatten und mit dem Ausfall formell ihren Beistandspflichten gegenüber den Lehensherren nach gekommen waren. Die Eidgenossen jedenfalls liessen die Garnison in ihrem Rücken unbehelligt; sie hatten auch gar nicht „ir büchsen und gezüg“ für eine Belagerung mitgeführt. Das zeigt, dass der Feldzug tatsächlich nur „eine streifreis“, also ein Beutezug war. Aus der Tschudi-Chronik wird deutlich, dass bereits in den vorhergehenden Wintern solche Feldzüge ins Sarganserland unternommen worden waren. Viel dürfte dort also nicht mehr zu holen gewesen sein; die Bevölkerung war die Raubzüge bereits gewohnt und hatte Massnahmen zur Sicherung ihres Viehs ergriffen. ${ }^{18}$ Die Eidgenossen rückten vor und erreichten den Hauptort Mels am 25. Februar 1446. Von hier aus schrieb der Luzerner Hauptmann Cloos an seine Obrigkeit einen der seltenen Briefe aus dem Feld. In seinem Schreiben betont er, dass die Leute nicht gerne warten würden, bis die lokale Bevölkerung ihnen die Treueeide schwören wollten. Sie wollten losschlagen. Gleichwohl war es für den langfristigen Erfolg des Kriegszuges vonnöten, die neue Situation durch die Bevollmächtigten der lokalen Gemeinden und der Amtsträger bestätigen zu lassen. ${ }^{19}$ Cloos summierte den bisherigen Verlauf des Feldzuges und gab die Absichten der Hauptleute weiter. Der Brief ist als Bericht an die Obrigkeit abgefasst und enthält keine Unsicherheiten oder Fragen. Der Kriegszug ging von Mels aus auf dem linken Ufer des Rheins weiter und dann setzten die Eidgenossen über den Rhein und lagerten am Triesenberg, in der Nähe der Truppen, die der berüchtigte Feldherr Hans von Rechberg im Auftrag des österreichischen Vogtes Ulrich von Matsch zusammengezogen hatte, um einen Gegenschlag vorzubereiten. Diese Truppen setzten sich vor allem aus den Aufgeboten der Stadt Feldkirch und dem Hinterland der Stadt zusammen. Seit dem Vorjahr standen hier mehr oder weniger ständig Leute unter Waffen. Davon haben wir ausgezeichnete Quellen, nämlich Soldquittungen Einzelner oder kleiner Gruppen. So bestätigte beispielsweise Rudi Zettel, am 2. März 1446, im Auftrag der Stadt Feldkirch dem österreichischen Vogt Ulrich von Matsch während eines Monats um sieben Gulden mit seinem Pferd gedient zu haben. ${ }^{20}$

Am Triesenberg hätten die Appenzeller die Eidgenossen verstärken sollen. Weil sie nicht kamen, zogen sich die Eidgenossen wieder über den Rhein nach Mels zurück; vor allem, weil die Streitmacht des Hans von Rechberg stetig wuchs. Als die Eidgenossen am

und wollten an die letzi und wurden der vigenden 16 erstochen und 2 gefangen.

18 Siehe Speich, H.: Netzwerke, S. 213.

19 StALU, 231/3390: Brief vom 8. März 1446 an Luzern, siehe Abbildung in Speich, Heinrich: Geschichtsfreunde. Neujahrsblätter des Historischen Vereins des Kantons St. Gallen 155, 2015, S. 145-147. Zu den Verhandlungen: Und uf fritag frü, so hant die herren von Pfeffers ir botschaft zu uns getan und mit uns geret von ir gotshus lüten, das wir ir schonen und si nit schlagen, so wetent si mit inen reden, dz si uns ze willen standen. Also hant wir inen geantwurt, wellent si uns sweren als ander, so wellent wir si ufnemen. Also wend si uns uf sanstag fruo antwurten; also werbendt ander berglüt ouch desglich. (...) Antwurten wir inen: wölten si üt mit uns reden von iemen, wölten wir gern lossen, doch dz sis kurz machte, wan wir wölte sölichs nüt lang warten, wir wölten uf unser figent ziechen und wölten si schatgen mit der hilf gotts an lib und an guot, als fer als wir möchten. Und sölicher antwurt warten wir untz uf sunentag frue und sind die unsern noch al früsch; me ze mal nüt me. Vgl. Speich, H.: Netzwerke, S. 208-221.

20 LA Vorarlberg, Urk 7667. Weitere zeitgenössische Soldquittungen siehe ebenda, Urk 7666, 7668, 7669 und 7670 . 
5. März in Mels hörten, dass sich die 4.000 Kämpfer Rechbergs in Ragaz zur Schlacht bereit machen würden, handelten sie. Im zweiten Brief von Hauptmann Cloos an seine Obrigkeit sind die Vorgänge um die Schlacht aus erster Hand beschrieben. ${ }^{21}$ Dieser zweite Bericht vom 8. März 1446 sowie der Bericht des Berner Hauptmanns Hans Wanner liefern zahlreiche Details zur Schlacht und zur Beute. Besonders die erbeuteten Fahnen und Feuerwaffen wurden detailliert aufgelistet. ${ }^{22}$ Cloos berichtet zudem nach Hause, die Eidgenossen hätten bei der Schlacht nur neun Mann verloren, eine Aussage, die durch die Jahrzeitbücher deutlich widerlegt wird. Davon später mehr. Cloos schätzt die gegnerischen Verluste auf über 300, das dürfte der tatsächlichen Zahl recht nahe kommen. Allein aus Feldkirch sind 42 Gefallene in den Jahrzeitbüchern verbürgt.

Diesmal berichtet Cloos nicht nur. Es tritt zu Tage, dass der Feldzug auf eine solche Schlacht nicht ausgerichtet war, und auf einen solchen Sieg schon gar nicht. Nur zwei Tage nach der Schlacht beklagt sich der Hauptmann bei seiner Obrigkeit in Luzern bitterlich, dass Sie wegen mangelnder Ausrüstung die umliegenden Festungen nicht angreifen könnten und statt dessen untätig in Mels liegen würden und wegen der hohen Preise nichts zu essen hätten. ${ }^{23}$ Cloos schliesst seinen Brief mit zwei eindringlichen Bitten: Lieben gnedigen Herren wir bitten üch flislich und ernstlich, $d z$ ir an sechen desse grossy not und hunger und unser armuot und $d z$ wir an der frömde sind und helfent üns umm gelt und dess fil wan lützel hilft üns nüt [...]. Die zweite Bitte ist die um Rat, wie sie sich weiter verhalten sollten. Cloos und die anderen Hauptleute hatten offenbar keine Ahnung, was sie weiter tun sollten. Ihre Leute wollten möglichst beutebeladen wieder abziehen, was auch nach der Schlacht von Ragaz geschah. ${ }^{24}$ Die Eidgenossen nutzten ganz offensichtlich den Sieg nicht, um eine Herrschaft zu etablieren. Deshalb zogen sie wieder ab. Zweihundert Glarner blieben noch im Land, aber als zwei Wochen später immer noch keine Verstärkung in Sicht war, zogen auch sie wieder ab.

21 Zweiter Brief von Hauptmann Cloos: StALU, 231/3391. Am Ausführlichsten zum Schlachtverlauf selbst äusserte sich der Bericht des Berner Hauptmanns Hans Wanner, welcher in der Chronik des Bendicht Tschachtlan (Zentralbibliothek Zürich, Ms A 120) eingearbeitet wurde. Dazu Ladner, Pascal: Tschachtlans Bilderchronik. Kommentar zur Faksimile-Ausgabe der Handschrift Ms A 120 der Zentralbibliothek Zürich. Luzern 1990, S. 398-401 (Textedition).

22 Jucker, Michael: Rauben, Plündern, Brandschatzen. Kriegs- und Fehdepraxis im Spannungsfeld von Recht, Ökonomie und Symbolik. In: Fehdeführung im spätmittelalterlichen Reich. Zwischen adeliger Handlungslogik und territorialer Verdichtung. Hg. von J. Eulenstein - Ch. Reinle - M. Rothmann. Studien und Texte zur Geistes und Sozialgeschichte des Mittelalters 7. Affalterbach 2013, S. 261-284.

23 StALU, 231/3391: Und ligen da und rouwen und het gern für genomen die vestÿ erübret und hant aber lützel ze essen und kein gelt und muessen alli ding um zwei gelt koufen und sind die ünser fast unwilig hie ze beliben wan sy nit ze essen hant $[\ldots]$.

24 Gemäss Tschudi bereits kurz nach dem Abzug der Eidgenossen, den er auf den 4. April datiert. In den übrigen Quellen ist kein genaues Abzugsdatum genannt. Sicher ist, dass österreichische Truppen schon bald danach im Thurgau und in Rapperswil aktiv wurden und dass der Graf im Herbst 1446 die Herrschaft wieder inne hatte. 


\section{Chronikalische Überlieferung}

Die österreichfreundliche Chronik des Twinger von Königshofen kommentierte lakonisch: und also entsausent inen die Schwitzer und zugent wider uss dem oberland und nam die herrschaft das Land wider in. ${ }^{25}$ Die kleine Toggenburger Chronik fasste die Schlacht von Ragaz zusammen mit den Worten: Die Schwitzer überfielent den Adel by der nacht, da si maindtend sicher zu sin. ${ }^{26}$ Sehr ausführlich und trotzdem verhalten kritisch zum Feldzug äusserte sich Hans Fründ: Da nu die im Land sahent, dass si nit trostes an inen hattent, und liessen hinder inen ire habe, und warent vor und nach arm lüte. Während Fründ Kritik an den Glarnern äussert, nimmt der Glarner Tschudi diese in seiner Chronik wieder zurück und führt den Misserfolg auf andere Gründe zurück. Weiter äussern sich die Chronisten Schilling, Brennwald und Schodoler ebenfalls zur Schlacht, sie fehlt in keiner der Chroniken des späten 15. und des 16. Jahrhunderts, obwohl die Schlacht selbst in der Grösse und Tragweite eher gering war. ${ }^{27}$ Dafür gibt es eine plausible Erklärung: es war die letzte grössere Auseinandersetzung im Alten Zürichkrieg, vor dem ersten erfolgreichen Waffenstillstand im Juni 1446. Somit wurden die Ereignisse nicht gleich vom nächsten Feldzug überrollt und die Erinnerung konnte auch schriftlich etwas ausführlicher gestaltet werden.

Normalerweise entstanden die Chroniktexte zur älteren Schweizer Geschichte schichtenweise, die Geschichten wucherten. Das ist hier nicht der Fall: die Chronisten hatten ihre spezifischen Vorgänger und Detailquellen, die Darstellungen sind aber weitgehend übereinstimmend. Gleichwohl ergänzte vor allem Tschudi die Ereignisse um Details, wie er dies bei fast allen Feldzügen tat. Aus seiner Feder stammen vor allem Orte und Personennamen, die er plausibel, aber nicht überprüfbar ergänzte.

\section{Schlachtengedenken}

Die Eidgenossen beschlossen kurz nach der Schlacht, dass der Schlachttag des 6. März es ist der Fridolinstag, Namenstag des Patrons der Glarner, die das grösste Kontingent in die Schlacht geführt hatten - als allgemeiner Schlachtengedenktag der Eidgenossen überall gefeiert werden sollte. Ein Blick in die Jahrzeitenbücher des Länderortes Schwyz zeigt, dass dies tatsächlich umgesetzt wurde. Das Jahrzeitbuch der Heligkreuzkirche Lachen in der March entstand beispielsweise erst um ca. 1520, enthielt aber unter dem 6. März die entsprechenden Schlachtengedenken, hier mit einem Schwerpunkt auf den Italienkriegen des frühen 16. Jahrhunderts. Der Text wurde eingeleitet mit der Präambel

25 Variante der Königshofen Chronik in Cod. Sang. 630, S. 298-299. Die Handschrift gehörte im 16. Jahrhundert Aegidius Tschudi. Dazu Scarpatetti, Beat Matthias von: Die Handschriften der Stiftsbibliothek St. Gallen. Bd. 1: Abt. IV, Codices 547-669. Hagiographica, Historica, Geographica, 8.-18. Jahrhundert. Wiesbaden 2003, S. 240-241.

26 Kleine Toggenburger Chroniken. Mit Beilagen und Erörterungen. Hg. von G. Scherrer. St. Gallen 1874, S. 51.

27 Heinrich Brennwalds Schweizerchronik. Hg. von R. Luginbühl. Quellen zur Schweizer Geschichte. Neue Folge I. Abteilung Chroniken. Bd. II. Heinrich Brennwalds Schweizerchronik II. Basel 1910, S. 167-168. 


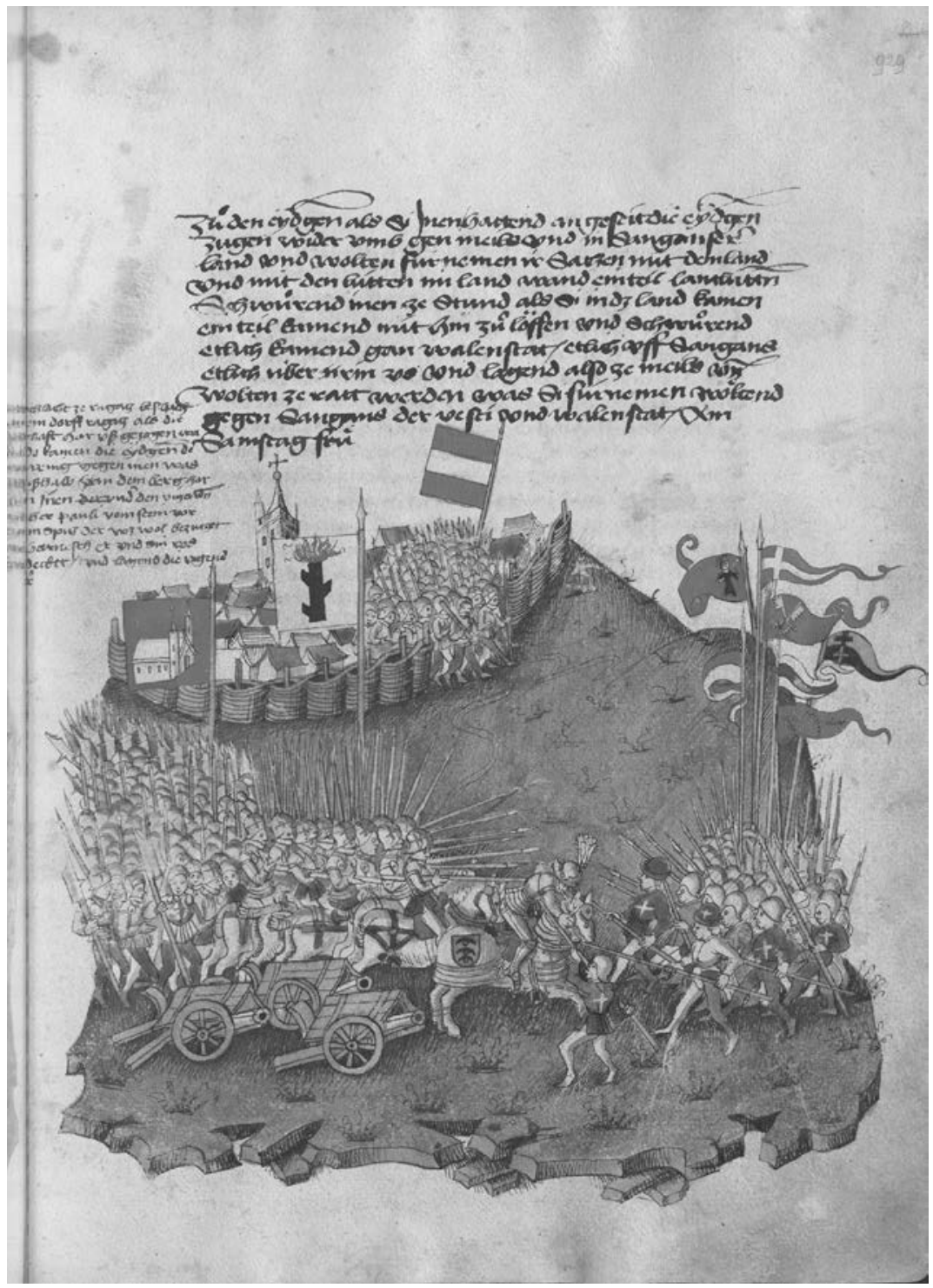

Abb. Die Schlacht bei Ragaz 1446 in der Chronik des Bendicht Tschachtlan (1470)

(Zentralbibliothek Zürich, Ms A 120, S. 929).

Disen tag hend gmeine landtlüth uffgenommen wie eins helgen apostlentag zfiren von wegen einer grossen schlacht, so im jar 1446 zuo Ratatz gschach. Man soll och uff gemelten tag jarzit halten aller deren, so ynn unserer landtlüten dienst und kriegen sind umkon. ${ }^{28}$ Wie der Text in die

28 Die Jahrzeitbücher des Kantons Schwyz. Bd. 1. Das Jahrzeitbuch der Pfarrkirche St. Martin, Schwyz. Bearb. von F. Auf der Maur. Schwyz 1999, S. 125 zu fol. 45v, bzw. Bd. 2. Das Jahrzeitbuch der Pfarrkirche Hl. Kreuz, Lachen. Bearb. von F. Auf der Maur, Schwyz 2001, S. 125. 
Jahrzeitbücher geriet, wird aus dem älteren Jahrzeitbuch der Hauptkirche von Schwyz deutlich, der den öffentlichen Charakter des Gedenkens unterstreicht. ${ }^{29}$ Zählt man die Toten in den Schwyzer Jahrzeitbüchern zusammen, sind es rund dreissig, die bei Ragaz verlorn. Weitere sind in Obwalder- oder Glarner Zahrzeitbüchern überliefert. Das widerspricht natürlich den Schilderungen Tschudis, der grossspurig behauptete, es kamend ouch an disem gefächt von den eidtgnossen umb vij man und nit mer, aber es ward ein michler tiel wund. ${ }^{30}$ Die Verwundeten, welche nicht wieder genasen, wurden in der Regel ebenfalls unter die Gefallenen der Schlacht gezählt und ihr Jahrtag wurde damit der ihrer tödlichen Verwundung. In der Stadt Luzern trieb die Verklärung des Fridolinstages noch weitere Blüten. Der Tag wurde zum Bestandteil der Fasnacht und bis heute kursiert in Luzern der übergrosse Bruder Fritschi als Personifizierung der Fasnacht. ${ }^{31}$

\section{Gerichtliches Nachspiel}

Nicht nur in Chronistik und Jahrzeiten schlug sich der Feldzug in den Quellen nieder. Auch in Gerichtsakten und Wirtschaftsquellen wurden die Auswirkungen sichtbar. In Vorarlberg beispielsweise zeigt eine Urkunde von 1456, dass damals von der Herrschaft hohe Kredite aufgenommen wurden, um das enorme Heer von 4.000 bis 5.000 Soldaten in der Kürze aufzustellen. So hatten allein die Grafen von Werdenberg-Sargans im März 1446 einen Kredit von 4600 Gulden dafür aufgenommen. Die Verzinsung machte ihnen noch zehn Jahre nach Kriegsende zu schaffen. ${ }^{32}$ In Gerichtsakten des Vorarlbergs wurde ebenfalls noch weiter in den alten Wunden gestochert: so berichtete Benedikt Bilgeri in der Geschichte Vorarlbergs, dass die Feldkircher geklagt hätten, weil sie nicht zur Verteidung ihres Landes, sondern für einen Feldzug gegen die Eidgenossen im Sarganserland aufgeboten worden wären. Das Kloster Pfäfers wiederum hätte wegen seiner Kontaktnahme mit den Eidgenossen während des Feldzuges die Habsburger entschädigen müssen. Die letzten beiden Gerichtsfälle lassen sich aus den Quellen allerdings nicht erhärten, es handelt sich

29 Ittem es soll mengklichen ewenklich zu wüssen sin, das wir, der lanndtamman und die lantlütte zu Schwytz unnd annder unnser getrüwen lieben Eydtgenossen, von behebung unsser alten ewigenn und geschwornen pünden unnd unnsser getrüwen Eydtgnoschaft wägen zu grossen, hertten, schwären, ouch tödtlichen kriegen leider kommen sind [...]. Und darumb so haben wyer [...] uffgenommen und gesetzt denselben sannt Frydlis tag ewenklich zu vyren, bys das man das heilig ampt volbringt, und also uff demselben tag, der frommen, nodtfesten byderben lütten aller jarrzyt zu began, so dan von den unnsseren, unnd von den annderen, unsseren getrüwen lieben Eydtgnossen, de in disen unnseren grossen kriegen vor unnd nach verlorn hanndt unnd also durch alles unnssers lanndt uff den tag getrüwlich unnd ewenklich geben uss und von unnsers gemeinen lanndts stüren unnd bruchen 18 pfund pfennig unnsers lanndtz wärung gott unnd unsser lieben frouwenn zu eren, dem wirdigen lieben heiligen sannt Fridlin, durch iro aller und aller glöübigen seelenheyll willen, also järlich zu teylen und zu gäben in yetliche unnsser lüthkillchen zu Schwytz [...]. Die Jahrzeitbücher. Bd. 1. Bearb. von F. Auf der Maur, S. 124.

30 Aegidius Tschudi, Chronicon Helveticum. Bd. 12, S. 421.

31 Vgl. Egloff, Gregor: Art. Fritschi. In: Historisches Lexikon der Schweiz. Bd. 4. Basel 2005, S. 840-841.

32 Vorarlberger Landesarchiv (Hohenems, Reichsgrafschaft), Urk. 8105. Zum Vergleich die Kriegsaufwendungen der Stadt Basel 1446 von total 10‘815 lb pf. bei Gesamtausgaben von 30‘511 lb pf. nach Rosen, Josef: Kriegsausgaben im Spätmittelalter. Der militärische Aufwand in Basel 1360-1535. In: ders.: Finanzgeschichte Basels im späten Mittelalter. Gesammelte Beiträge 1971-1987. Stuttgart 1989, S. 457-484, S. 469. 
um Konjekturen von lokalen Historiographen. Als Siegerin in diesem kleinen Krieg darf trotz verlorener Schlacht die Herrschaft Österreich gelten: Die Eidgenossen hatten es nicht geschafft, sich während des Feldzuges genügend Rückhalt für eine dauerhafte Besetzung zu schaffen. Der Chronist Tschudi unterstellt den Beteiligten zwar, mit dieser Intention vorgegangen zu sein. Die Organisation des Feldzuges und das Vorgehen während der Besetzung sprechen allerdings dagegen. Als Österreich das Land im April 1446 wieder eingenommen hatte, sassen die österreichisch gesinnten Parteigänger sofort wieder fest im Sattel. Die Herrschaft hatte sogar genug Mittel, um ihren Parteigängern, die Schaden erlitten hatten, grosszügige Entschädigungen zukommen zu lassen. Das geht jedenfalls aus einer Urkunde vom 24. April 1446 hervor, als Erni Maiger vom Hubmeister zu Feldkirch eine Nutzniessung über 80 Pfund Pfennig zur Tilgung von Kriegsschäden erhielt. ${ }^{33}$ Die Eidgenossen ihrerseits vergassen nicht, dass ihnen die Grafen von Werdenberg-Sargans und die Freiherren von Brandis in Vaduz feindlich gesinnt waren. Nach der erneuten Eroberung des Sarganserlandes im Jahre 1460 im Rahmen der Thurgauer Wirren wurde jedenfalls eine gemeine Herrschaft errichtet, die bis 1798 Bestand hatte. ${ }^{34}$

\section{Zusammenfassung}

Aus den Ostschweizer Städten und Länderorten sowie aus Vorarlberg sind aus dem Umfeld des Feldzuges von 1446 zahlreiche Quellengattungen fassbar, die sonst rein im städtischen Konfliktbereich, selten im adligen Kontext und bis dahin kaum im ländlichen Bereich bekannt sind.

Für die prominente Verarbeitung des Geschehens in der eidgenössischen Historiographie spricht noch ein weiteres Moment: in der bereits im späten Mittelalter auf Föderalismus und Proporz ausgerichteten Grosserzählung zum Werden der Eidgenossenschaft eignete sich die Schlacht von Ragaz vorzüglich. Die Zürcher waren zwar mit Hans von Rechberg und einigen Knechten auf österreichischer Seite präsent. Aber es war bedeutend einfacher, wieder einmal einen Sieg gegen die bösen Erbfeinde aus dem Hause Habsburg feiern zu können, als sich mit der eigenen jüngsten Vergangenheit mit den äusserst blutigen Auseinandersetzungen zwischen Zürich und den Innerschweizer Orten befassen zu müssen. Die Kämpfe des Alten Zürichkrieges fanden im nationalen Narrativ bevorzugt dort einen Platz, wo sie sich gegen äussere Feinde richteten. Interessanterweise wurden auch die Augenzeugenberichte des Berner Hauptmanns und die Chronik des Hans Fründ in der Folge historiographisch ausgewertet: Der Bericht von Wanner in der Tschachtlan-Chronik, die wiederum Vorbild der wirkmächtigen Berner Chronik des Diebold Schilling wurde. Fründs Beschreibung wurde durch Tschudi ergänzt und zur

33 Vorarlberger Landesarchiv (ohne Provenienz), Urk 1633.

$34 \mathrm{Zu}$ den Ereignissen 1460 und den Auswirkungen auf die einzelnen Herrschaften im St. Galler Oberland siehe Die Rechtsquellen des Kantons St. Gallen. Dritter Teil: Landschaften und Landständen. Bd. 2. Die Rechtsquellen des Sarganserlandes. Bearb. von S. Malamud - P. Sutter. Sammlung Schweizerischer Rechtsquellen 14. Aarau 2013, S. LIII-LXXVI. 
Meistererzählung ausgeschmückt. Die einzige überlieferte Abschrift des Manuskriptes von Fründ befand sich nämlich später im Besitz von Aegidius Tschudi.

Kleiner Feldzug - grosse Wirkung. Der sogenannte Ragazer Feldzug am Ende des Alten Zürichkrieges ist trotz seiner lokalen Wirkung in zahlreichen Quellengattungen präsent. Weil nachher keine bedeutenderen Auseinandersetzungen mehr erfolgten, geriet der Konflikt nicht in Vergessenheit, sondern wurde aufgearbeitet und mit neuen Bedeutungsebenen versehen auf die Stufe einzelörtischer, später nationaler Geschichte gehoben. Gerade den sonst quellenmässig schwach aufgestellten Länderorten der Eidgenossenschaft verhalf der Konflikt zur Verschriftlichung weiterer Bereiche. Die Herausforderungen im Alten Zürichkrieg ermöglichten es den Länderorten, im Bereich von Schriftlichkeit, Diplomatie und Historiographie mit den Städteorten gleich zu ziehen und diese stellenweise sogar in den Schatten zu stellen.

\section{Nach demm und wir üch jetz geschriben hant von der getat. Vojenské rády, kroniky, zprávy a listy z bitevního pole. Prameny k ragazskému tažení 1446 za tzv. Staré curyšské války}

V únoru 1446 vytáhlo 1100 členů Spř́íseženstva z Bernu, Lucernu, Uri, Schwyzu, Unterwaldenu, Zugu a Glarusu do sousedního Sarganserlandu, aby zakročili proti Habsburkům a jejich vazalovi hraběti Jindřichovi IX. z Werdenberg-Sargansu. Tzv. Ragazské tažení mělo z pohledu členů Spříseženstva hladký průběh. Zvítězili v bitvě u Ragazu, ale později byli ze země opět vytlačeni. Bylo to poslední velké tažení v rámci tzv. Staré curyšské války (1440-1446).

Z tažení se dochovalo mnoho pramenů, které příkladně ukazují, jaké typické texty mohly vzniknout v souvislosti s válečnictvím v 15. století. Kromě toho jsou poprvé doložitelné prameny z venkovských komunit (Länderorte) Spříseženstva, které svědčí o intenzivním písemnictví během války.

Příprava je patrná z protokolů jednání zemských zástupců. Přímo z bojiště se dochovaly dva listy luzernského hejtmana Cloose, v nichž podal zprávu a také naléhavě prosil o posily a peníze. Na habsburské straně se dochovaly kvitance pro žoldnéře a dlužní listy. Po vítězné bitvě u Ragazu byla tato událost začleněna do historiografie Spř́íseženstva. Máme k dispozici ještě dvě soudobé zprávy k bitvě: zprávu bernského hejtmana pro svou vlastní vrchnost a kroniku schwyzského zemského písaře Hanse Fründa. Jelikož bylo ragazské tažení poslední v řadě bitev tzv. Staré curyšské války, 6. březen byl stanoven jako výroční den i pro jiné bitvy zmíněné války. Jména padlých byla zapsána ke svátku sv. Fridolína, glaruského patrona, do knih anniversárií (Jahrzeitbücher). Na základě uvedených pramenů bylo tažení začleněno do kronikářství Spříseženstva, které z něho učinilo úspěch celého spolku. 